\title{
From Evidence-based Medicine to Personalized Medicine
}

\author{
Suk-Hwan Lee \\ Department of Surgery, Kyung Hee University School of Medicine, Seoul, Korea
}

\section{See Article on Page 259-264}

Over the last two decades, many studies have shown the role of the adjuvant chemotherapy in stage III colon cancer [1-8]. With the introduction of excellent cytotoxic agents such as oxaliplatin and irinotecan, the median survival of even stag IV disease has been prolonged by more than 24 months. Based on the National Comprehensive Cancer Network (NCCN) guideline and the recently published Korean guideline, the oxaliplatin-based regimen has been the preferred regimen for stage III colon cancer over a single-agent regimen such as the capecitabine or 5-fluorouracil/ leucovorin (FL) regimen. However, some concerns exist regarding the toxicity and the efficacy in adjuvant chemotherapy for stage III colon cancer. Because adjuvant chemotherapy means largely prophylactic chemotherapy rather than therapeutic chemotherapy like palliative chemotherapy, the efficacy of adjuvant chemotherapy should be balanced with its possible side effects, such as peripheral neuropathy or febrile neutropenia. For many years, the ideal duration of adjuvant chemotherapy has been under evaluation. Particularly with regard to the cumulative neuropathic adverse effects of oxaliplatin, a further decrease in the treatment duration is warranted. The SAFFA study showed no differences in term of overall survival between six months of a bolus FL regimen or three months of a protracted FL regimen [3]. However, a shorter adjuvant treatment duration is currently under evaluation in the IDEA program, which includes 12,000 patients from six ongoing trials [9].

Recently, the elderly population has grown remarkably worldwide. South Korea is also one of the countries with a fast-growing

Correspondence to: Suk-Hwan Lee, M.D.

Department of Surgery, Kyung Hee University Hostpital at Gangdong, Kyunghee University School of Medicine, 892 Dongnam-ro, Gangdong-gu, Seoul 134-727, Korea

Tel: +82-2-440-6134, Fax: +82-2-440-6295

E-mail: leeshdr@khu.ac.kr

(C) 2012 The Korean Society of Coloproctology

This is an open-access article distributed under the terms of the Creative Commons Attribution NonCommercial License (http://creativecommons.org/licenses/by-nc/3.0) which permits unrestricted noncommercial use, distribution, and reproduction in any medium, provided the original work is properly cited. elderly population. The subgroup analysis of the NSABP C-07 trial showed a trend towards better disease-free survival in patients younger than age 70 (hazard ratio, $0.80 ; 95 \%$ confidence interval, 0.68 to $0.95 ; \mathrm{P}=0.013$ ), but no positive effect was evident in older patients with the addition of oxaliplatin to the FL regimen [10]. Even the NCCN guideline recommended that the oxaliplatinbased regimen not be used for elderly patients older than 70 years. Conversely, capecitabine showed a constant efficacy even in elderly patients [11].

Lymph node metastasis is the single most important prognostic factor in colon cancer. It also determines the necessity of adjuvant chemotherapy in colon cancer. In the 7th American Joint Committee on Cancer tumor-node-metastasis staging system [12], the 5 -year survival of stage IIIA colon cancer was even better than that of stage IIA colon cancer, which raised the concerns about the necessity of and ideal regimen for adjuvant chemotherapy in stage IIIA colon cancer. In this issue of the Journal of the Korean Society of Coloproctology, an article entitled "Oncologic Outcome of Stage IIIA Colon Cancer According to Chemotherapeutic Regimen" dealt with this specific concern. Although the study was a single-institution-based retrospective review and included a relatively small number of patients $(n=131)$ to draw a concrete conclusion, single-agent-based chemotherapeutic regimens (capecitabine or FL) showed 5-year disease free and overall survival rates similar to those of oxaliplatin-based regimens.

In stage IIIA colon cancer, the depth of invasion is limited to the muscularis propria, and lymph node metastases are minimal (up to 6 metastatic lymph nodes). Among the eight patients who did not receive the adjuvant chemotherapy, three patients had recurrences during the follow-up, which suggested that adjuvant chemotherapy has a role even in stage IIIA colon cancer.

This study suggested the need for further clinical trials in Korea to obtain solid evidence for this specific condition. The incidence of stage IIIA disease is very low, so it takes a long time to collect the patients to identify effective chemotherapeutic agents as in this paper (from 1995 to 2008, only 131 patients were collected). During that time in Korea, the governmental insurance policies changed continuously, so it was hard to measure the real value of the different regimens of adjuvant chemotherapeutic agents. However, this study provides possible clues for individualized or per- 
sonalized chemotherapy. In the near future, I hope the Korean Society of Coloproctology may have a role in addressing this specific question.

\section{REFERENCES}

1. Andre T, Boni C, Mounedji-Boudiaf L, Navarro M, Tabernero J, Hickish T, et al. Oxaliplatin, fluorouracil, and leucovorin as adjuvant treatment for colon cancer. N Engl J Med 2004;350:2343-51.

2. Andre T, Boni C, Navarro M, Tabernero J, Hickish T, Topham C, et al. Improved overall survival with oxaliplatin, fluorouracil, and leucovorin as adjuvant treatment in stage II or III colon cancer in the MOSAIC trial. J Clin Oncol 2009;27:3109-16.

3. Chau I, Norman AR, Cunningham D, Tait D, Ross PJ, Iveson T, et al. A randomised comparison between 6 months of bolus fluorouracil/leucovorin and 12 weeks of protracted venous infusion fluorouracil as adjuvant treatment in colorectal cancer. Ann Oncol 2005;16:549-57.

4. de Gramont A, Hubbard J, Shi Q, O’Connell MJ, Buyse M, Benedetti J, et al. Association between disease-free survival and overall survival when survival is prolonged after recurrence in patients receiving cytotoxic adjuvant therapy for colon cancer: simulations based on the 20,800 patient ACCENT data set. J Clin Oncol 2010; 28:460-5.

5. Haller DG, Tabernero J, Maroun J, de Braud F, Price T, Van Cutsem E, et al. Capecitabine plus oxaliplatin compared with fluorouracil and folinic acid as adjuvant therapy for stage III colon can- cer. J Clin Oncol 2011;29:1465-71.

6. Mochizuki I, Takiuchi H, Ikejiri K, Nakamoto Y, Kinugasa Y, Takagane A, et al. Safety of UFT/LV and S-1 as adjuvant therapy for stage III colon cancer in phase III trial: ACTS-CC trial. Br J Cancer 2012;106:1268-73.

7. Twelves C, Wong A, Nowacki MP, Abt M, Burris H 3rd, Carrato A, et al. Capecitabine as adjuvant treatment for stage III colon cancer. N Engl J Med 2005;352:2696-704.

8. Van Cutsem E, Labianca R, Bodoky G, Barone C, Aranda E, Nordlinger B, et al. Randomized phase III trial comparing biweekly infusional fluorouracil/leucovorin alone or with irinotecan in the adjuvant treatment of stage III colon cancer: PETACC-3. J Clin Oncol 2009;27:3117-25.

9. Stein A, Hiemer S, Schmoll HJ. Adjuvant therapy for early colon cancer: current status. Drugs 2011;71:2257-75.

10. Yothers G, O’Connell MJ, Allegra CJ, Kuebler JP, Colangelo LH, Petrelli NJ, et al. Oxaliplatin as adjuvant therapy for colon cancer: updated results of NSABP C-07 trial, including survival and subset analyses. J Clin Oncol 2011;29:3768-74.

11. Twelves C, Scheithauer W, McKendrick J, Seitz JF, Van Hazel G, Wong A, et al. Capecitabine versus 5-fluorouracil/folinic acid as adjuvant therapy for stage III colon cancer: final results from the $\mathrm{X}$-ACT trial with analysis by age and preliminary evidence of a pharmacodynamic marker of efficacy. Ann Oncol 2012;23:1190-7.

12. Edge SB, Byrd DR, Compton CC, Fritz AG, Greene FL, Trotti A. AJCC cancer staging manual. 7th ed. New York: Springer; 2010. 